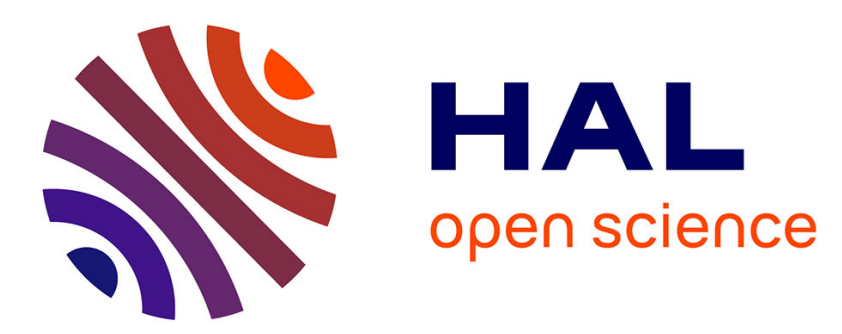

\title{
Composés métalliques de nickel et gallium sur GaAs par codéposition en ultravide
}

\author{
Y. Ballini, A. Guivarc'H, J. Caulet, A. Chomette, B. Le Merdy
}

\section{To cite this version:}

Y. Ballini, A. Guivarc'H, J. Caulet, A. Chomette, B. Le Merdy. Composés métalliques de nickel et gallium sur GaAs par codéposition en ultravide. Revue de Physique Appliquée, 1989, 24 (1), pp.71-78. 10.1051/rphysap:0198900240107100 . jpa-00246031

\section{HAL Id: jpa-00246031 https://hal.science/jpa-00246031}

Submitted on 1 Jan 1989

HAL is a multi-disciplinary open access archive for the deposit and dissemination of scientific research documents, whether they are published or not. The documents may come from teaching and research institutions in France or abroad, or from public or private research centers.
L'archive ouverte pluridisciplinaire HAL, est destinée au dépôt et à la diffusion de documents scientifiques de niveau recherche, publiés ou non, émanant des établissements d'enseignement et de recherche français ou étrangers, des laboratoires publics ou privés. 
Classification

Physics Abstracts

$68.55-73.40 \mathrm{~N}-73.60 \mathrm{D}-79.60$

\title{
Composés métalliques de nickel et gallium sur GaAs par codéposition en ultravide
}

\author{
Y. Ballini, A. Guivarc'h, J. Caulet, A. Chomette et B. Le Merdy \\ CNET LAB/OCM/MPA, BP 40, F-22301, Lannion Cedex, France
}

(Reçu le $1^{\text {er }}$ juillet 1988, accepté le 7 octobre 1988)

\begin{abstract}
Résumé. - Nous avons étudié la codéposition de couches minces de nickel et de gallium sur des substrats de GaAs (001) et GaAs (111) dans la perspective d'une épitaxie d'un composé métallique, si possible thermodynamiquement stable. Les codépôts ont été réalisés à température ambiante dans un bâti d'épitaxie par jets moléculaires équipé d'un canon à électrons pour l'évaporation du nickel. Quatre techniques ont été mises en œuvre pour contrôler la composition ; deux ex situ : la rétrodiffusion d'ions $\mathrm{He}^{+}$de $2 \mathrm{MeV}$ (RBS), qui nous a servi de référence, et l'analyse $\mathrm{X}$ dans un microscope électronique à balayage; et deux in situ : les spectroscopies de photo-émission électronique X et UV (XPS et UPS). En UPS nous avons utilisé d'une part l'évolution de la forme de la bande de valence en fonction de la composition, d'autre part le déplacement chimique du doublet $\mathrm{Ga}_{3 \mathrm{~d}}$ par rapport au niveau de Fermi dû au transfert de charge entre Ni et Ga. Les couches déposées ont été caractérisées par diffraction $\mathrm{X}$ sur goniomètre à poudre $\theta-2 \theta$ équipé d'un monochromateur arrière. Les caractérisations électriques $\left(R_{\square}\right)$ et par canalisation d'ions $\mathrm{He}^{+}\left(\chi_{\min }\right)$ montrent que la résistivité est minimale et la canalisation maximale pour les couches les mieux texturées, correspondant aux composés définis $\mathrm{NiGa}$ et $\mathrm{Ni}_{2} \mathrm{Ga}_{3}$. Ces composés ont des conductivités du même ordre de grandeur que les siliciures de métaux de transition.
\end{abstract}

\begin{abstract}
We have studied the codeposition of thin layers of nickel and gallium on (001) and (111) GaAs substrates in order to epitaxy metallic compounds, thermodynamically stable if possible, on GaAs. The codepositions were made in a molecular beam epitaxy (MBE) growth chamber equipped with a Ni electron beam evaporation source. Four techniques have been used to control the composition : two ex situ, Rutherford backscattering (RBS) with $2 \mathrm{MeV} \mathrm{He}{ }^{+}$ions (which served as a reference) and X-ray analysis in a scanning electron microscope, and two in situ : X-ray and ultraviolet photoemission spectroscopies (XPS and UPS). The UPS controls have used the evolution of the valence band shape as a function of composition and the chemical shift of the $\mathrm{Ga}_{3 \mathrm{~d}}$ doublet due to the charge transfer between $\mathrm{Ni}$ and $\mathrm{Ga}$. The deposited layers have been characterized by X-ray diffraction in a $\theta-2 \theta$ powder goniometer equipped with a monochromator. The electrical characterization $\left(R_{\square}\right)$ and the characterization of $\mathrm{He}^{+}$channeling $\left(\chi_{\min }\right)$ show that the resistivity and the $\chi_{\min }$ are the lowest for the best textured layers, which correspond to the compounds $\mathrm{NiGa}$ and $\mathrm{Ni}_{2} \mathrm{Ga}_{3}$. These compounds have conductivities of the same order as transition metals silicides.
\end{abstract}

\section{Introduction.}

L'intégration de plus en plus poussée de composants électroniques et optoélectroniques à base de semiconducteurs III-V passe par l'étape essentielle de la réalisation de contacts de bonne conductivité, thermodynamiquement stables et latéralement homogènes. L'objectif est d'obtenir pour les semiconducteurs III-V ce qui a déjà été réalisé avec le silicium et les siliciures : épitaxie d'une couche métallique sur le semiconducteur et reprise d'épitaxie du semiconducteur sur cette couche métallique (à l'exemple du système $\left.\mathrm{Si} / \mathrm{CoSi}_{2} / \mathrm{Si}\right)$. Pour cela, compte tenu de la réactivité des éléments métalliques purs sur les semiconducteurs III-V, il faut trouver des composés métalliques susceptibles d'adopter une relation épitaxiale avec le substrat semiconducteur et thermodynamiquement stables avec lui [1]. C'est dans ce but qu'ont déjà été étudiés les systèmes $\mathrm{AuGa}_{2} / \mathrm{GaSb}$ [2], $\mathrm{RhGa} / \mathrm{GaAs}$ [3], $\alpha-\mathrm{Rh}_{2} \mathrm{As} / \mathrm{GaAs}$ [4], $\mathrm{NiGa} / \mathrm{GaAs}[5,6]$, et $\mathrm{NiAl} / \mathrm{GaAs}$ [7].

Notre laboratoire a fait le choix expérimental $[8$, $3,4,5]$ d'une codéposition en ultravide à partir de sources séparées pour réaliser de tels composés 
métalliques. Dans le cadre d'une étude plus générale menée sur le système ternaire $\mathrm{Ni}-\mathrm{Ga}-\mathrm{As}$ [9] et les interdiffusions en phase solide sur les contacts $\mathrm{Ni} / \mathrm{GaAs}[10]$, nous avons pu constater que parmi les divers composés du nickel dans ces systèmes, trois sont thermodynamiquement stables avec GaAs : $\mathrm{NiGa}, \mathrm{Ni}_{2} \mathrm{Ga}_{3}$, NiAs. Nous avons de plus précisé les relations cristallographiques existant entre les composés de structures cubique et pseudocubique (structure hexagonale telle que $c / a \simeq \sqrt{3} / \sqrt{2}$ ), et les substrats de GaAs (001) et (111) [10]. Les principaux composés binaires $\mathrm{Ni}_{x} \mathrm{Ga}_{1-x}$ sont cubiques ou pseudocubiques et présentent des possibilités d'épitaxie avec GaAs en raison de désaccords de mailles relativement faibles (au maximum 2,1\%, dans le cas de $\mathrm{NiGa} / \mathrm{GaAs}$ ) (cf. Tabl.).

C'est ce qui nous a conduit à examiner les possibilités offertes par les composés métalliques binaires de $\mathrm{Ni}$ et $\mathrm{Ga}$ pour une inter-épitaxie avec GaAs. Dans cet article nous présentons nos premiers résultats concernant les composés $\mathrm{Ni}_{x} \mathrm{Ga}_{1-x}$ obtenus par codéposition de $\mathrm{Ni}$ et $\mathrm{Ga}$ sur $\mathrm{GaAs}(001)$ et GaAs (111).

\section{Fabrication des composés métalliques.}

Les codépositions ont été effectuées dans un bâti ultravide d'Epitaxie par Jets Moléculaires (EJM, alias MBE: Molecular Beam Epitaxy) RIBER MBE 2300 RD sur des substrats GaAs (001) et GaAs (111) semi-isolants. La chambre de dépôts est équipée d'une source d'évaporation de métaux réfractaires par canon à électrons AIRCO TEMESCAL dotée d'un cache amovible. Le flux de métal ainsi produit est mesuré et régulé à l'aide d'un oscillateur à quartz. Pour faciliter les comparaisons entre couches nous avons toujours utilisé le même flux (correspondant à $0,08 \mathrm{~nm} / \mathrm{s}$, sauf pour quelques couches pour lesquelles nous avons réduit le flux à $0,03 \mathrm{~nm} / \mathrm{s}$ ) et nous avons toujours déposé la même quantité de nickel (l'équivalent de $28 \mathrm{~nm}$ de métal pur).

Le flux de gallium est issu d'une cellule classique RIBER CBN 125 et contrôlé par régulation de la température de celle-ci. Une composition visée $\mathrm{Ni}_{x} \mathrm{Ga}_{1-x}$ est obtenue en choisissant en conséquence le rapport des flux de $\mathrm{Ni}$ et $\mathrm{Ga}$; soit, comme nous avons travaillé à flux de $\mathrm{Ni}$ constant, en choisissant en conséquence la température de la cellule de $\mathrm{Ga}$. L'épaisseur des couches déposées varie ainsi d'environ $58 \mathrm{~nm}$ pour $\mathrm{Ni}_{3} \mathrm{Ga}_{2}$ à environ $89 \mathrm{~nm}$ pour $\mathrm{Ni}_{2} \mathrm{Ga}_{3}$.

Sur chaque porte-échantillons en molybdène (« molybloc » de $0,55 \mathrm{~nm}$ ) nous avons toujours collé (à l'indium) trois substrats : GaAs (001) semi-isolant, GaAs (111) semi-isolant et silicium passivé par oxydation en surface (ce dernier servant de substrat de référence chimiquement inerte, amorphe, ne contenant pas d'élément constitutif des couches et adapté à l'analyse par RBS par sa masse relativement faible).

Les substrats GaAs ont été préparés suivant la méthode usuelle en EJM, puis introduits dans la chambre de dépôts où ils sont désoxydés à $600^{\circ} \mathrm{C}$ sous flux d'As 4 . La température des substrats est ensuite ramenée à température ambiante $\left(20^{\circ} \mathrm{C}\right)$ et y est maintenue pendant les codépositions pour éviter toute interaction directe des atomes de nickel avec ceux des substrats GaAs.

Quatre techniques ont été mises en œuvre pour contrôler la composition; deux in situ, dans une chambre d'analyse adjacente à la chambre de dépôts, juste après déposition des couches : les spectroscopies de photoémission électronique $X$ et UV (XPS, UPS : X-ray or Ultraviolet Photoemission Spectroscopy); et deux ex situ: la rétrodiffusion d'ions (RBS : Rutherford Back Scattering) et l'analyse X dans un microscope électronique à balayage.

La RBS a été effectuée avec un faisceau incident d'ions $\mathrm{He}^{+}$de $2 \mathrm{MeV}$ sur les composés déposés sur substrat $\mathrm{Si}$ passivé; elle nous a servi de référence pour les autres techniques. Le spectre reporté sur la figure $1 \mathrm{a}$, caractéristique de ce genre d'analyse, montre que les contributions de $\mathrm{Ni}$ et $\mathrm{Ga}$ sont bien séparées de celle du substrat de silicium. Un programme de calcul très simple permet de séparer les contributions de nickel et de gallium qui se chevauchent légèrement lorsque l'on analyse des couches

Tableau. - Données cristallographiques (le réseau cubique de NiGa suivant l'axe 〈111〉est pseudohexagonal).

[Crystallographic data (the cubic lattice of $\mathrm{NiGa}$ along $\langle 111\rangle$ is pseudo-hexagonal).]

\begin{tabular}{|c|c|c|c|c|c|c|}
\hline & Structure & Paramètres (nm) & $c / a$ & $\begin{array}{l}\text { Désaccords de } \\
\text { sur GaAs (111) sur }\end{array}$ & $\begin{array}{l}\text { maille } \\
\text { GaAs (001) }\end{array}$ & $\begin{array}{l}\text { Nbre de molécules } \\
\left(\text { en } 10^{22} \mathrm{~cm}^{-3}\right)\end{array}$ \\
\hline $\mathrm{Ni}_{3} \mathrm{Ga}_{2}$ & hex. $\mathrm{P}_{3} / \mathrm{mmc}$ & $\begin{array}{l}a=0,3992 \\
c=0,4960\end{array}$ & 1,242 & $-0,1 \%$ & $+0,9 \%$ & 1,453 \\
\hline $\mathrm{NiGa}$ & cub. Pm3m & $a=0,2886$ & 1,225 & $+2,1 \%$ & $+2,1 \%$ & 4,160 \\
\hline $\mathrm{Ni}_{2} \mathrm{Ga}_{3}$ & hex. P3ml & $\begin{array}{l}a=0,4055 \\
c=0,4889\end{array}$ & 1,206 & $+1,4 \%$ & $+1,0 \%$ & 1,437 \\
\hline
\end{tabular}




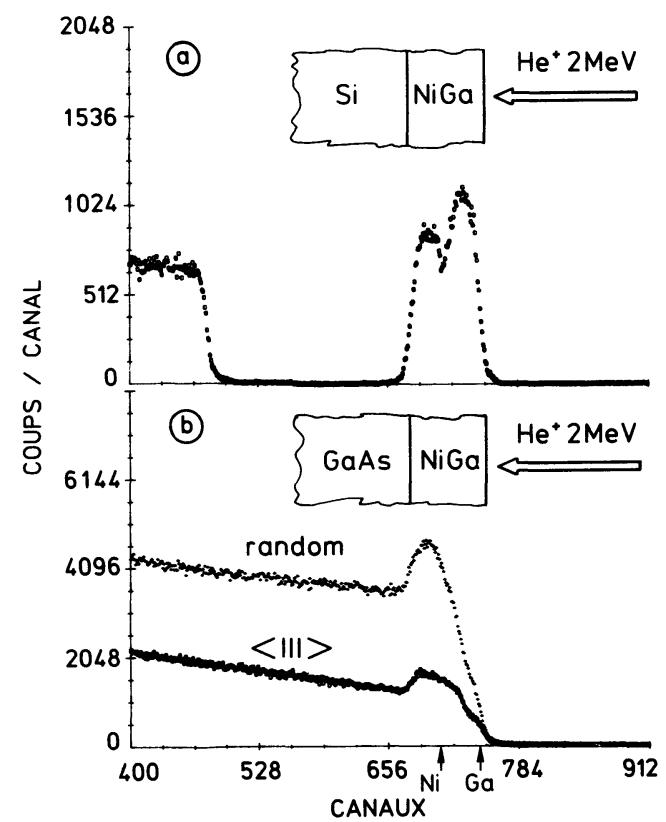

Fig. 1. - Spectres de rétrodiffusion d'ions $\mathrm{He}^{+}(\mathrm{RBS}) \mathrm{de}$ $\mathrm{NiGa}$ : a) sur Si passivé ; b) sur GaAs (111).

[ $\mathrm{He}^{+}$ion $\mathrm{RBS}$ spectra of $\mathrm{NiGa}$ : a) on passivated $\mathrm{Si}$; b) on (111) GaAs.]

de ces épaisseurs à l'aide de faisceau d'ions $\mathrm{He}^{+}$de $2 \mathrm{MeV}$. La comparaison de leur surface conduit directement au rapport des concentrations atomiques (égal à $1 \pm 0,06$ pour l'échantillon considéré).

Il est à noter que ces valeurs ont été vérifiées d'une part en analysant ce même échantillon à l'aide d'ions $\mathrm{He}^{+}$de 2,5 MeV (les contributions de nickel et de gallium sont alors nettement séparées) et d'autre part en effectuant des simulations numériques beaucoup plus complexes [10] sur les spectres correspondant aux codépôts sur GaAs. Les variations de composition entre les diverses déterminations entrent dans l'erreur indiquée.

L'analyse $\mathrm{X}$ a porté sur le rayonnement $\mathrm{X}$ émis par ces mêmes composés déposés sur substrat $\mathrm{Si}$ sous l'action d'un faisceau d'électrons de $20 \mathrm{keV}$. Les raies $\mathrm{X}$ analysées sont les raies $K \alpha_{1}$ du nickel et du gallium (respectivement à $7478 \mathrm{eV}$ et à $9251 \mathrm{eV}$ ). Les résultats de l'analyse ont été interprétés en considérant les couches comme milieu semi-infini ; c'est-à-dire en négligeant l'influence du substrat, bien que celui-ci soit visible compte tenu de l'épaisseur relativement faible des couches (quelques dizaines de nanomètres). Cette approximation favorise légèrement $\mathrm{Ga}$ par rapport à $\mathrm{Ni}$, mais facilite grandement l'interprétation.

Sur la figure 2 nous comparons les déterminations de compositions par RBS et par analyse X. La concordance est satisfaisante compte tenu de l'approximation indiquée ci-dessus, montrant que l'une ou l'autre technique peuvent être indifféremment utilisées.

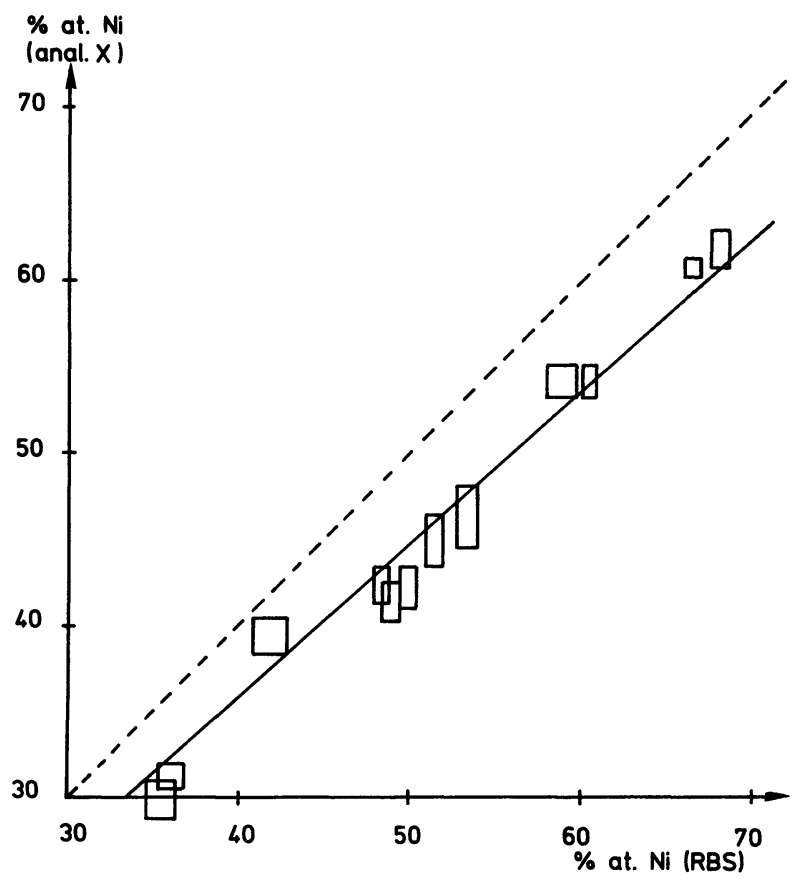

Fig. 2. - Comparaison des déterminations de compositions $\mathrm{Ni}_{x} \mathrm{Ga}_{1-x}$ par rétrodiffusion d'ions (RBS) et par analyse $\mathrm{X}$ en microscope électronique à balayage.

[Comparison of $\mathrm{Ni}_{x} \mathrm{Ga}_{1-x}$ compositions determinated by RBS and by X-ray analysis in scanning electron microscope.]

\section{Spectroscopies de photoémission électronique $\mathrm{X}$ et UV.}

La chambre d'analyse de notre bâti d'EJM est équipée d'un analyseur d'électrons semi-dispersif RIBER MAC 2, d'une source X RIBER CX 700 (AlK $\alpha$ et $\mathrm{MgK} \alpha$, sans monochromateur) et d'une source UV LEYBOLD-HERAEUS UVS 10/35 (utilisée en hélium, également sans monochromateur). Les programmes d'acquisition et de traitement des données ont été mis au point dans notre laboratoire.

L'XPS permet de vérifier la désoxydation des substrats $\mathrm{GaAs}$ avant codéposition et de déterminer la composition des couches déposées. Les mesures ont été effectuées sur la plupart des échantillons quel que soit le type de substrat. Nous avons principalement utilisé la partie du spectre XPS présentant les énergies de liaison inférieures à $80 \mathrm{eV}$, partie qui comporte trois raies voisines en énergie et représentatives des trois principaux éléments : $\mathrm{Ni}_{3 \mathrm{p}}(\mathrm{a} 68 \mathrm{eV})$, $\mathrm{As}_{3 \mathrm{~d}}(\mathrm{a} 44 \mathrm{eV})$ et $\mathrm{Ga}_{3 \mathrm{~d}}(\mathrm{à} 19 \mathrm{eV})$. Les raies relatives à l'arsenic n'apparaissent qu'en cas de pollution involontaire ou en cas d'interaction avec le substrat; la figure 3 montre un exemple d'un tel spectre.

Les composés étudiés étant tous de composition chimique similaire, la profondeur d'échappement des photoélectrons sera très voisine d'échantillon en échantillon pour chaque raie. Dans ces conditions l'XPS, seulement semi-quantitative ordinairement, 


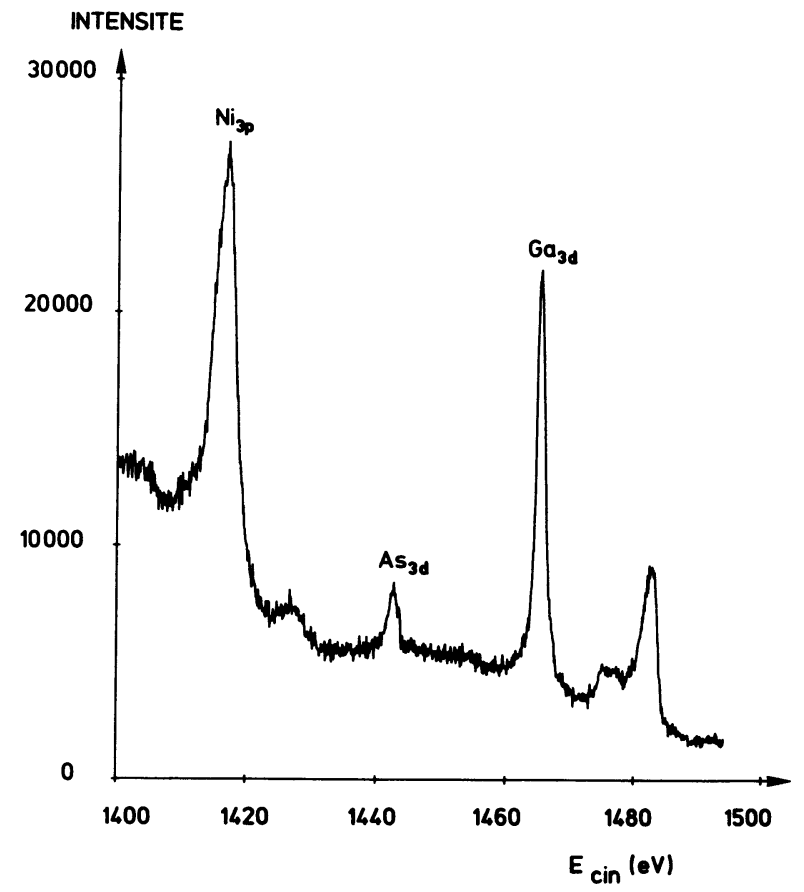

Fig. 3. - Spectre de photoémission électronique $\mathrm{X}$ en AlK $\alpha$ montrant les raies d'énergie de liaison inférieure à $80 \mathrm{eV}$; ce spectre est un exemple de couche $\mathrm{Ni}_{x} \mathrm{Ga}_{1-x}$ présentant une légère pollution d'arsenic révélée par la présence du pic $\mathrm{As}_{3 \mathrm{~d}}$.

[AlK $\alpha$ XPS spectrum at binding energies lower than $80 \mathrm{eV}$; this spectrum is an example of $\mathrm{Ni}_{x} \mathrm{Ga}_{1-x}$ in which a small arsenic pollution is revealed by the presence of the $\mathrm{As}_{3 \mathrm{~d}}$ peak.]

permet d'obtenir des mesures relatives quantitatives: le rapport des proportions de nickel et de gallium est pratiquement proportionnel au rapport des intensités de raies $\mathrm{Ni}_{3 \mathrm{p}}$ et $\mathrm{Ga}_{3 \mathrm{~d}}$, soit aussi au rapport des hauteurs de pics $\mathrm{Ni}_{3 \mathrm{p}}$ et $\mathrm{Ga}_{3 \mathrm{~d}}$.

On peut effectivement constater sur la figure 4 que ce rapport de hauteurs de pics varie à peu près linéairement en fonction de la composition déterminée a posteriori par RBS; la dispersion observée peut s'expliquer pour partie par une dispersion inhérente à la XPS elle-même et pour partie par une inhomogénéité des couches (les analyses par XPS et par RBS ne sont pas effectuées sur les mêmes points et la composition varie légèrement d'un bord à l'autre des couches).

Cette méthode nous a permis de disposer d'une évaluation in situ de la composition des couches déposées assez simple et rapide de mise en œuvre (par la mesure du rapport des hauteurs de pics $\mathrm{Ni}_{3 \mathrm{p}}$ et $\mathrm{Ga}_{3 \mathrm{~d}}$ et la lecture du rapport des proportions $\mathrm{Ni} / \mathrm{Ga}$ qui lui correspond sur la droite représentée Fig. 4).

En UPS nous avons surtout utilisé la raie $\mathrm{He}_{\mathrm{II} \alpha}$ qui, avec une énergie d'excitation de $40,81 \mathrm{eV}$, nous donne :

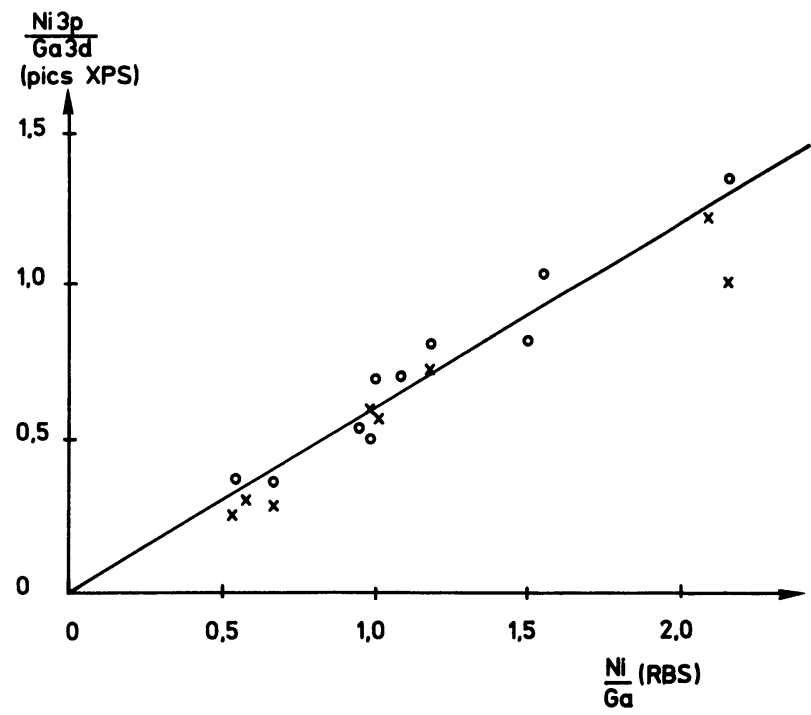

Fig. 4. - Rapport des hauteurs de pics de photoémission $\mathrm{X} \mathrm{Ni}_{3 \mathrm{p}}$ et $\mathrm{Ga}_{3 \mathrm{~d}}$ en fonction du rapport de compositions $\mathrm{Ni} / \mathrm{Ga}$ déduit de la rétrodiffusion d'ions (RBS).

[Ratio of XPS peak heights of $\mathrm{Ni}_{3 \mathrm{p}}$ and $\mathrm{Ga}_{3 \mathrm{~d}}$ as a function of the ratio of compositions $\mathrm{Ni} / \mathrm{Ga}$ as deduced from $\mathrm{RBS}$.]

(i) un spectre de bande qui est une image pratiquement sans déformation de la densité d'états dans la bande de valence en dessous du niveau de Fermi,

(ii) un doublet $\mathrm{Ga}_{3 \mathrm{~d}}$ convenablement résolu (énergies de liaison moyennes par rapport au niveau de Fermi de $19,2 \mathrm{eV}$ pour $\mathrm{Ga}_{3 \mathrm{~d} 3 / 2}$ et $18,8 \mathrm{eV}$ pour $\mathrm{Ga}_{3 \mathrm{~d} 5 / 2}$ ); ce doublet n'est pas visible à l'aide de la raie $\mathrm{He}_{\mathrm{I}}$, d'énergie trop faible $(21 \mathrm{eV})$ et ne peut être résolu en XPS.

Les raies satellites $\mathrm{He}_{\mathrm{II} \beta}$ et $\mathrm{He}_{\mathrm{II} \gamma}$ sont suffisamment décalées en énergie par rapport à $\mathrm{He}_{\mathrm{II} \alpha}$ (respectivement de $+7,56$ et $+10,20 \mathrm{eV}$ ) pour que leurs contributions au spectre UPS global soient clairement séparées ; le doublet $\mathrm{Ga}_{3 \mathrm{~d}}$ est d'ailleurs exploitable en $\mathrm{He}_{\mathrm{II} \beta}$.

Les raies excitatrices $\mathrm{He}_{\mathrm{II}}$ sont suffisamment étroites pour que la résolution expérimentale globale ne soit limitée que par les possibilités de l'analyseur d'électrons. Une étude préalable sur une couche d'argent fraîchement déposée nous a permis de mesurer une résolution limite de $0,12 \mathrm{eV}$ pour le MAC 2. Par ailleurs notre système d'acquisition nous impose un pas élémentaire d'échantillonnage en énergie de $30 \mathrm{meV}$.

Les niveaux de Fermi de tous les composés métalliques examinés coïncident (à un pas près en énergie) et nous avons utilisé ce niveau commun comme niveau de référence des énergies de liaison.

La position du niveau $\mathrm{Ga}_{3 d}$ en énergie est fonction du transfert de charge entre les atomes de nickel et de gallium dépendant de l'environnement chimique du gallium dans le composé $\mathrm{Ni}_{x} \mathrm{Ga}_{1-x}$. Nous avons décelé en UPS un déplacement chimique pour le 
niveau $\mathrm{Ga}_{3 \mathrm{~d}}$ en fonction de la composition; ce déplacement est d'environ $300 \mathrm{meV}$ entre les compositions extrêmes étudiées $\left(\mathrm{Ni}_{2 / 3} \mathrm{Ga}_{1 / 3}\right.$ et $\left.\mathrm{Ni}_{1 / 3} \mathrm{Ga}_{2 / 3}\right)$, valeur trop faible pour être décelée en XPS sans l'utilisation d'une source $\mathrm{X}$ monochromatisée.

En principe ce déplacement chimique devrait être observé en quelques valeurs discrètes correspondant aux composés définis du système $\mathrm{Ni}-\mathrm{Ga}: \mathrm{Ni}_{3} \mathrm{Ga}$, $\mathrm{Ni}_{5} \mathrm{Ga}_{3}, \mathrm{Ni}_{3} \mathrm{Ga}_{2}, \mathrm{NiGa}, \mathrm{Ni}_{3} \mathrm{Ga}_{4}, \mathrm{Ni}_{2} \mathrm{Ga}_{3}^{*}, \mathrm{NiGa}_{4}$. Dans cette étude préliminaire, où de plus certains codépôts conduisent à des mélanges de phases, nous n'avons pas pu établir clairement une telle correspondance (cf. Fig. 6a).

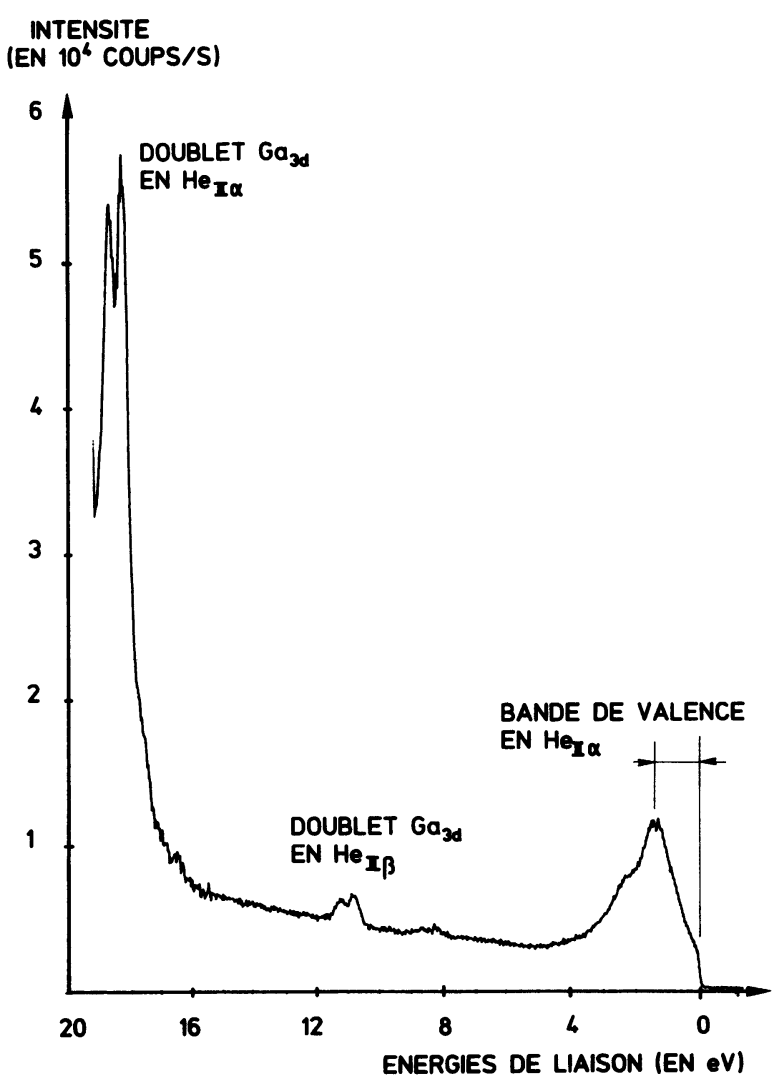

Fig. 5. - Spectre de photoémission électronique de $\mathrm{NiGa}$ sous excitation UV $\mathrm{He}_{\text {II }}$ montrant le doublet $\mathrm{Ga}_{3 \mathrm{~d}}$ en $\mathrm{He}_{\text {II } \alpha}$ et $\mathrm{He}_{\mathrm{II} \beta}$ et la bande de valence en $\mathrm{He}_{\mathrm{II} \alpha}$; la caractéristique de cette bande utilisée dans cette étude est la position énergétique du maximum de la densité d'états par rapport au niveau de Fermi.

[ $\mathrm{He}_{\mathrm{II}}$ UPS spectrum of $\mathrm{NiGa}$ showing the $\mathrm{Ga}_{3 \mathrm{~d}}$ doublet excited by $\mathrm{He}_{I I \alpha}$ and $\mathrm{He}_{I I \beta}$ and the $\mathrm{He}_{I I \alpha}$ valence band ; the band characteristic used in this study is the energetic position of the maximum of the density of states with respect to the Fermi level.]

La densité d'états électroniques dans la bande de valence en fonction de l'énergie est une fonction assez sensible de la composition $\mathrm{Ni}_{x} \mathrm{Ga}_{1-x}$. De plus, dans le cas d'une composition intermédiaire entre deux composés définis, nous ne percevons qu'un effet moyen au niveau de la bande de valence. Nous nous sommes attachés à une caractéristique simple de cette densité d'états : la position du maximum par rapport au niveau de Fermi (cf. Fig. 5). Nous avons observé que cette position varie continûment avec la composition moyenne et, après étalonnage, fournit une détermination assez fiable de celle-ci (cf. Fig. 6b).

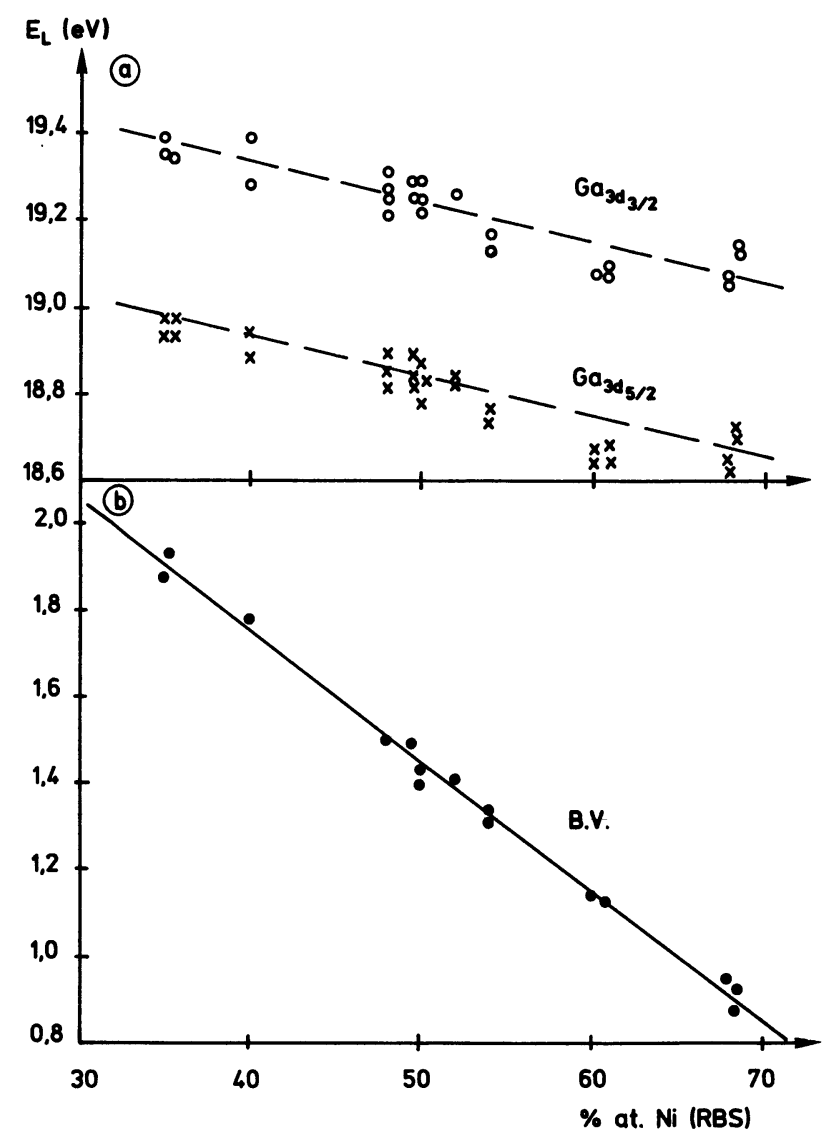

Fig. 6. - Photoémission électronique UV : déplacement chimique du doublet $\mathrm{Ga}_{3 \mathrm{~d}}$ et position du maximum de la bande de valence par rapport au niveau de Fermi en fonction de la composition déterminée par rétrodiffusion d'ions (RBS). O: $\mathrm{Ga}_{3 \mathrm{~d} 3 / 2} ; \times: \mathrm{Ga}_{3 \mathrm{~d} 5 / 2}$.

[UPS : chemical shift of the $\mathrm{Ga}_{3 \mathrm{~d}}$ doublet and position of the maximum of the density of states with respect to the Fermi level as the function of the composition determinated in RBS.]

Les dépôts sont effectués par séries de trois molyblocs successifs. Compte tenu de la difficulté de reproduire parfaitement les flux de $\mathrm{Ni}$ et $\mathrm{Ga}$ d'une série à l'autre, les caractérisations in situ nous ont permis de rectifier les rapports de flux au sein d'une série pour ajuster les compositions visées avec une assez bonne précision. 
Caractérisations électriques $\left(\boldsymbol{R}_{\square}\right)$ et par canalisation d'ions

Toutes les couches ont été caractérisées par la méthode des quatre pointes alignées (mesure de la résistance carrée $R_{\square}$ permettant la détermination de la conductivité) et par canalisation d'ions $\mathrm{He}^{+}$ rétrodiffusés; nous présentons en figure $1 \mathrm{~b}$ des spectres de rétrodiffusion obtenus sur substrat GaAs (111) suivant des directions incidentes du faisceau d'ions $\mathrm{He}^{+}$parallèles à l'axe $\langle 111\rangle$ du substrat (spectre canalisé $\langle 111\rangle$ ), et ne correspondant à aucun axe ou plan cristallographique (spectre « random »). On constate sur cette figure une diminution des contributions au spectre du nickel et du gallium lorsque le faisceau incident est canalisé. Le paramètre $\chi_{\min }$, défini comme étant le rapport des contributions de $\mathrm{Ni}$ ou de $\mathrm{Ga}$ sur les spectres canalisé et « random », est dans ce cas égal à 0,40 , valeur significative d'une relation épitaxiale déjà marquée par rapport au substrat.

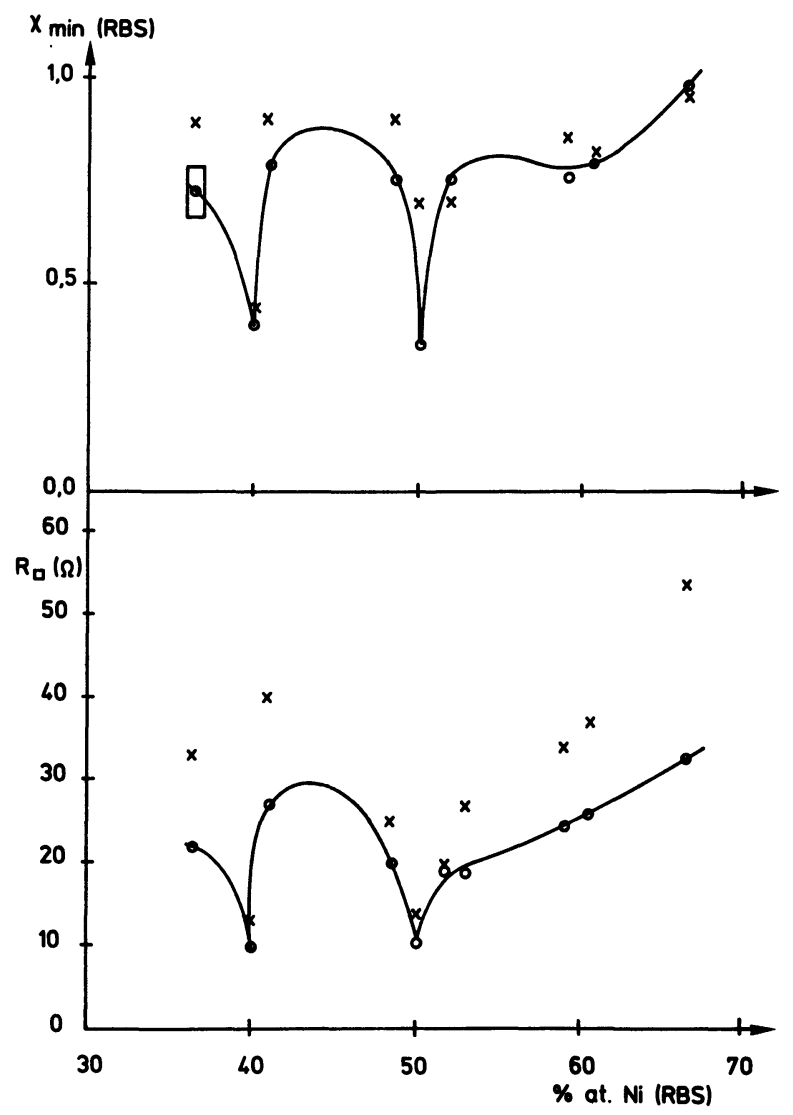

Fig. 7. - Résistance carrée $R_{\square}$ et rapport $\chi_{\min }$ de canalisation en fonction de la composition déterminée par rétrodiffusion d'ions (RBS). (Le rectangle sur le premier point est représentatif de la précision des mesures de $\left.\chi_{\min }\right)$. O : substrat GaAs (111); $\times$ : substrat GaAs $(001)$.

[Resistivity $\left(R_{\square}\right)$ and channeling ratio $\chi_{\min }$ as a function of composition determinated in RBS (the rectangle on the first point represents the precision of $\chi_{\min }$ measurements).]
Nous avons reporté sur une même figure (Fig. 7) le comportement de $R_{\square}$ et de $\chi_{\min }$ en fonction de la composition pour les composés déposés sur substrat GaAs (111) et sur substrat (001). Ces graphiques font apparaître clairement la singularité des couches dont la composition correspond assez précisément à $\mathrm{Ni}_{2} \mathrm{Ga}_{3}(40 \% \mathrm{Ni})$ et à $\mathrm{NiGa}(50 \% \mathrm{Ni})$. Pour ces deux compositions le phénomène de canalisation est très prononcé ( $\chi_{\min }$ de 0,4 contre 0,7 à 0,9 pour les autres compositions), comportement révélateur d'une couche bien texturée par rapport au substrat. Et c'est exactement pour les mêmes compositions que la résistivité est minimale, le $R_{\square}$ descendant jusqu'à $10 \Omega$ faisant de $\mathrm{Ni}_{2} \mathrm{Ga}_{3}$ et $\mathrm{NiGa}$ des composés aussi bons conducteurs que les siliciures de métaux de transition (sans traitement thermique ultérieur, $\rho \simeq 65 \mu \Omega \mathrm{cm}$ pour $\mathrm{NiGa}$ et $\rho \simeq 90 \mu \Omega \mathrm{cm}$ pour $\left.\mathrm{Ni}_{2} \mathrm{Ga}_{3}\right)$.

\section{Caractérisation par diffraction $\mathbf{X}$.}

Les couches déposées ont été caractérisées par diffraction $\mathrm{X}$ sur un goniomètre automatisé à poudre RIGAKU type CN 2155D5 équipé d'un monochromateur arrière de quartz. La longueur d'onde utilisée est $\mathrm{Cu} \mathrm{K} \alpha_{1}(\lambda=0,154051 \mathrm{~nm})$.

Une étude parallèle sur le système ternaire Ni-GaAs (synthèse directe de matériaux massifs) nous a permis de disposer des spectres de diffraction $\mathrm{X}$ de référence pour les divers composés de ce système [9]. Les caractérisations par diffraction $X$ ont toutes été effectuées sur substrat GaAs (111).

Les mouvements $\theta$ et $2 \theta$ peuvent être couplés ou pilotés séparément, de façon à pouvoir rattraper l'angle de coupe de substrat en ramenant la normale aux plans réticulaires (111) dans le plan diffractant $\mathrm{du}$ goniomètre.

Les couches polycristallines donnent certaines raies intenses du diagramme de poudre des composés, avec cependant une intensité assez faible compte tenu de la petite quantité de matériau diffractant.

Lorsqu'ils ne sont pas polycristallins, les composés étudiés ont tous une texture suivant la direction (111) du substrat. Le réglage préliminaire compensant l'angle de coupe des substrats (111) suffit pour nous donner les réflexions $(00 \ell)$ des couches déposées texturées.

Ces couches ne donnent que les raies déterminées par cette texture, l'intensité étant nettement plus élevée que pour les couches polycristallines non texturées.

Nous n'avons observé de couches très texturées que pour les compositions correspondant aux composés définis $\mathrm{Ni}_{2} \mathrm{Ga}_{3}, \mathrm{NiGa}$ et $\mathrm{Ni}_{3} \mathrm{Ga}_{2}$. Le comportement varie rapidement avec la composition $\mathrm{Ni}_{x} \mathrm{Ga}_{1-x}$; ainsi avec $37 \%$ ou $42 \%$ de $\mathrm{Ni}$ nous observons $\mathrm{Ni}_{2} \mathrm{Ga}_{3}$ polycristallin, seule la composition 


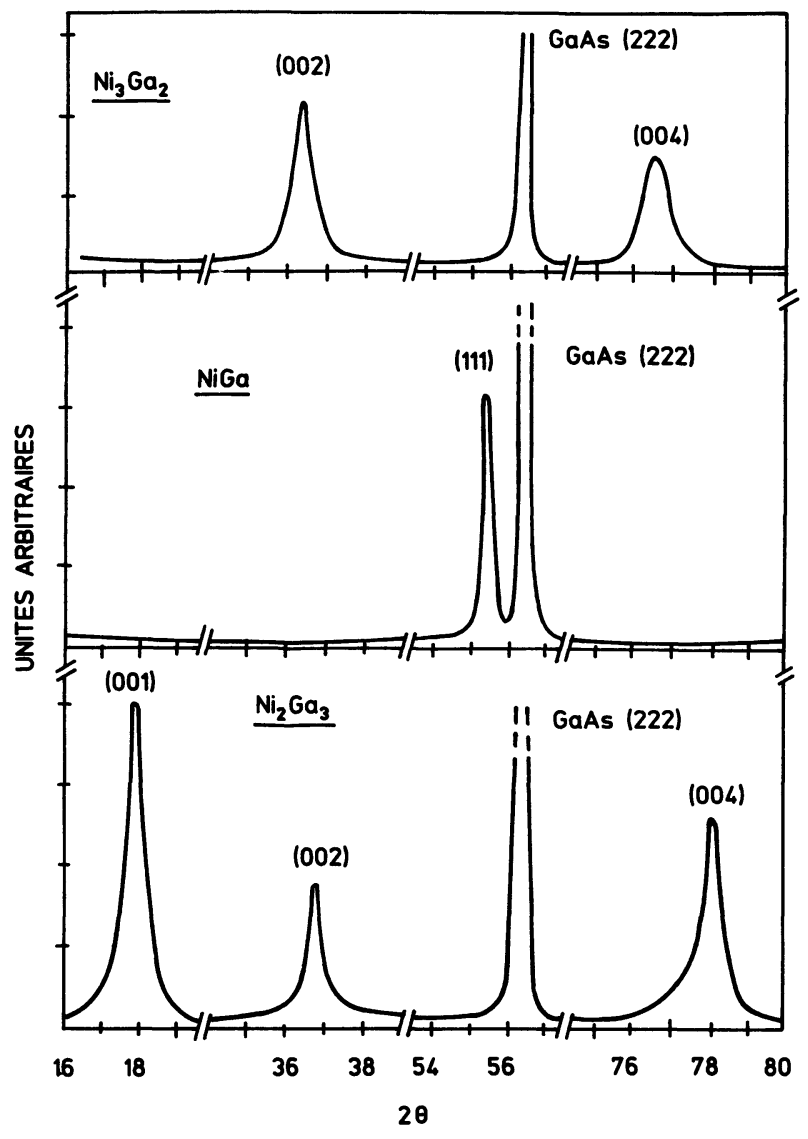

Fig. 8. - Spectres de diffraction $\mathrm{X}$ de $\mathrm{Ni}_{2} \mathrm{Ga}_{3}, \mathrm{NiGa}$ et $\mathrm{Ni}_{3} \mathrm{Ga}_{2}$.

[X-diffraction spectra of $\mathrm{Ni}_{2} \mathrm{Ga}_{3}, \mathrm{NiGa}$ and $\mathrm{Ni}_{3} \mathrm{Ga}_{2}$.]

$40 \%$ correspondant à une couche très texturée de $\mathrm{Ni}_{2} \mathrm{Ga}_{3}$, donnant également les plus faibles $\chi_{\min }$ en RBS et la meilleure conductivité (cf. Fig. 7).

Ce phénomène est moins pointu pour les composés voisins de $50 \%$ de $\mathrm{Ni}$, puisqu'une bonne texture $\mathrm{NiGa}$ est observée de $48 \%$ à $54 \%$, la couche la plus texturée correspondant à $50 \%$ et coïncidant avec le plus faible $\chi_{\min }$ et la meilleure conductivité (cf. Fig. 7).

Cette différence de comportement s'explique principalement par l'extension des domaines d'existence des phases $\mathrm{Ni}_{2} \mathrm{Ga}_{3}$ et $\mathrm{NiGa}$ (respectivement de $40 \%$ à $40,5 \%$ et de 45 à $53 \%$ de Ni) [11].

Pour les composés plus riches en $\mathrm{Ni}$ seule la composition $59 \%$ nous a donné une texture (le domaine d'existence de $\mathrm{Ni}_{3} \mathrm{Ga}_{2}$ est $57,7 \%$ à $60 \%$ ), bien que nous n'ayons pas observé de phénomène de canalisation particulier en RBS sur cet échantillon, ni de fléchissement dans la résistivité (cf. Fig. 7).

Il ressort de ces diverses caractérisations que lorsque le rapport des flux incidents correspond précisément à la composition des composés définis présentant des possibilités d'accord de maille avec les substrats GaAs, la qualité des épitaxies et la conductivité électrique des couches sont améliorées.

\section{Conclusion.}

Nous avons fabriqué des composés $\mathrm{Ni}_{x} \mathrm{Ga}_{1-x}$ en couche mince sur substrat GaAs (001) et (111) par codéposition en ultravide à partir de sources séparées de $\mathrm{Ni}$ et $\mathrm{Ga}$. Les couches obtenues sont d'autant mieux épitaxiées que le rapport des flux est proche de la stœchiométrie d'un composé défini présentant un désaccord de maille suffisamment faible avec le substrat GaAs. Cette stœchiométrie peut être vérifiée a posteriori ex situ grâce à la rétrodiffusion d'ions (RBS), à l'analyse $\mathrm{X}$ et à la diffraction des rayons $\mathrm{X}$; mais nous avons montré la possibilité d'une vérification immédiate in situ grâce à la photoémission électronique X ou UV (XPS et UPS), en utilisant des caractéristiques aisément accessibles des spectres XPS et UPS dont les variations avec la composition ont été calibrées à l'aide des méthodes ex situ.

Dans cette étude préliminaire nous avons toutefois rencontré des difficultés à maîtriser convenablement le flux de métal à partir d'un canon à électron ; de plus, par ses contraintes géométriques dans la chambre de dépôts, cette source de métal nous a provoqué des problèmes de pollution d'arsenic. Pour améliorer la qualité des épitaxies nous allons utiliser une cellule pour le nickel dont la position sera voisine des autres cellules de notre chambre de dépôts ; nous obtiendrons ainsi une vitesse de croissance mieux maîtrisée, plus faible, permettant en principe une croissance couche à couche.

Dans la perspective d'une reprise d'épitaxie du semiconducteur GaAs sur le composé métallique épitaxié, il nous faudra étudier la stabilité thermique des structures $\mathrm{NiGa} / \mathrm{GaAs}$ et $\mathrm{Ni}_{2} \mathrm{Ga}_{3} / \mathrm{GaAs}$ sous vide (pour déterminer une température qui soit à la fois assez basse pour assurer cette stabilité et assez élevée pour permettre une bonne épitaxie de GaAs). Les premiers éléments en ce sens tendent à faire de $\mathrm{NiGa}$ le meilleur candidat pour GaAs (001) ; par contre $\mathrm{Ni}_{2} \mathrm{Ga}_{3}$ sur substrat $\mathrm{GaAs}$ (111) présente la meilleure stabilité thermique et devrait donc permettre une température de réépitaxie plus élevée que pour les autres structures.

\section{Remerciements.}

Nous tenons à remercier nos collègues du CNET qui nous ont apporté leur aide pour cette étude, en particulier Jean-Pierre Mercier, Georges Dupas, Gilles Ropars et André Regreny. 


\section{Bibliographie}

[1] a) Tsai, C. T. and Williams, P. S., J. Mater. Res. 1 (1986) 352-360.

b) Beyers, R., Ki Bum Kim, Sinclair R., J. Appl. Phys. 61 (1987) 2195-2202.

[2] Lince, J. R. and Williams, R. S., J. Vac Sci. Technol. B 3 (1985) 1217-1220; Thin Solid Films 137 (1986) 251-265.

[3] Guivarc'H, A., Secoué, M. and Guénais, B., Appl. Phys. Lett. 52 (1988) 948-950.

[4] a) Secoute, M., Guénais, B. and Guivarc'H, A., Rev. Phys. Appl. 22 (1987) 845-850.

b) Guivarc'H, A., Secoué, M., GuÉnais, B., BaLLINI, Y., BADOZ, P. A. and ROSENCHER, E., J. Appl. Phys. 64 (1988) 683-687.

[5] GuivarC'H, A., Guérin, R. and Secoue, M., Electron. Lett. 23 (1987) 1004-1005.
[6] SANDS, T., Proc. 45th annual meet. Electron Microsc. Soc. Amer. (1987) 322-325.

[7] a) Sands, T., Appl. Phys. Lett. 52 (1988) 197-199.

b) SANDs, T., Harbison, J. P., Chan, W. K., Schwarz, S. A., Chang, C. C., Palmstrom, C. I. and Heramidas, V. G., Appl. Phys. Lett. 52 (1988) 1216-1218.

[8] Secoute, M., Thèse de l'Université de Rennes 1, $n^{\circ} 119$ (1987).

[9] Guérin, R. and Guivarc'H, A., soumis à J. Appl. Phys.

[10] Guivarc'h, A., Guérin, R., Caulet, J., PoudouLeC, A. and Fontenille, J., soumis à J. Appl. Phys.

[11] Feschotte, P. et Eggimann, P., J. Less-Common Met. 63 (1979) 15-30. 